\title{
Nonlinear structural analysis using integrated force method
}

\author{
N R B KRISHNAM RAJU and J NAGABHUSHANAM
}

Department of Aerospace Engineering, Indian Institute of Science, Bangalore 560012 , India

e-mail:\{balram,naga\}@aero.iisc.ernet.in

\begin{abstract}
Though the use of the integrated force method for linear investigations is well-recognised, no efforts were made to extend this method to nonlinear structural analysis. This paper presents the attempts to use this method for analysing nonlinear structures. General formulation of nonlinear structural analysis is given. Typically highly nonlinear bench-mark problems are considered. The characteristic matrices of the elements used in these problems are developed and later these structures are analysed. The results of the analysis are compared with the results of the displacement method. It has been demonstrated that the integrated force method is equally viable and efficient as compared to the displacement method.
\end{abstract}

Keywords. Finite element analysis; nonlinear analysis; force method.

\section{Introduction}

Force method in the pre-computer era was the popular analysis tool for civil, mechanical and aerospace engineering structures. This popularity can be attributed to its ability to determine accurate estimates of forces in the structure. During the formulative period of structural analysis by matrix methods, earnest research was directed to automate the force method, which includes the computer-assisted generation of compatibility conditions. This effort, by and large, was not fully successful (Robinson \& Haggenmacher 1971; Topou 1979; Kaneko et al 1983); redundant analysis being the main cause of the failure; it acted as the dominating road block in the path of force method automation.

A new formulation termed the Integrated Force Method (IFM) was proposed by Patnaik (1973) for the analysis of discrete and continuous systems. IFM is a force method of analysis which is independent of redundants and basis determinate structure of the classical force method. A variational functional has also been established (Patnaik 1986). The stationary condition of the functional yields the equilibrium equations, compatibility and natural boundary conditions. This method has been successfully used on many linear structures for their static behaviour such as skeletal frames (Patnaik \& Yadagiri 1989), plane stress (Nagabhushanam \& Srinivas 1991), plates and three dimensional problems (Kaljevic et al 1996b). Realizing its potential in structural analysis, it was further extended to consider investigations on dynamics (Patnaik \& Yadagiri 1976) and optimization (Patnaik et al 1996) of structures. 
Looking at the existing nonlinear structural analysis literature, almost all the investigation appear to use the displacement method except for a few (Oden \& Neighbors 1976) who use the force method (most of the time restricted to skeletal structures). This paper presents the extension of the integrated force method to study nonlinear structural problems. The scope of the paper is restricted to the analysis of the geometric nonlinear behaviour of the structure. A number of classical nonlinear beam type of structures are considered. They are analysed with IFM and their results are compared with those from the displacement solution. The studies demonstrate that IFM is an equally viable alternative method to the present very popular displacement method.

\section{Basic theory of integrated force method}

This section describes the development of basic theory of the integrated force method and presents its governing equations. A structure in the force method of analysis can be designated "Structure $(n, m)$ " where $(n, m)$ are the force and displacement degrees of freedom. The $n$ component force vector $\{\mathbf{F}\}$ must satisfy $m$ equilibrium equations along with $r=$ $(n-m)$ compatibility conditions. For a discrete structure, the equilibrium equations can be symbolized as

$$
[\mathbf{B}]\{\mathbf{F}\}=\{\mathbf{P}\} .
$$

The equilibrium equations represent the vectorial summation of the internal forces $\{\mathbf{F}\}$ to the external load $\{\mathbf{P}\}$ at the nodes of the finite element discretization. $\{\mathbf{F}\}$, the internal forces are the primal variables of equilibrium. The equilibrium matrix $[\mathbf{B}]$ is rectangular and sparse in nature and its generation is straightforward. The internal energy (IE) of the structure considering the nodal displacements $\{\mathbf{X}\}$ of the structure can be written as

$$
\mathrm{IE}=\frac{1}{2}\{\mathbf{X}\}^{T}\{\mathbf{P}\}=\frac{1}{2}\{\mathbf{X}\}^{T}[\mathbf{B}]\{\mathbf{F}\} .
$$

The internal energy of the structure can also be written considering the deformations of the elements as follows.

$$
\mathrm{IE}=\frac{1}{2}\{\mathbf{F}\}^{T}\{\boldsymbol{\beta}\}
$$

where $\{\boldsymbol{\beta}\}$ represents the vector of generalised internal deformations of the elements.

Considering (2) and (3), we can write

$$
\frac{1}{2}\{\mathbf{F}\}^{T}\left([\mathbf{B}]^{T}\{\mathbf{X}\}-\{\boldsymbol{\beta}\}\right)=0 .
$$

As $\{\mathbf{F}\}$ is not a null vector, it leads to displacement deformations relation

$$
[\mathbf{B}]^{T}\{\mathbf{X}\}-\{\boldsymbol{\beta}\}=0
$$

The deformation displacement relation represents $n$ deformations expressed in terms of $m$ displacements which leads to $(n-m)$ constraints on the deformations of the elements. The constraint on deformations are called compatibility conditions and are expressed through a compatibility matrix $[\mathbf{C}]$ and $\{\boldsymbol{\beta}\}$ which can be written as

$$
[\mathbf{C}]\{\boldsymbol{\beta}\}=0 \text {, }
$$


where $[\mathbf{C}]$ is called the compatibility matrix. Compatibility conditions can be further expressed in terms of primal variables $\{\mathbf{F}\}$, noting $\{\boldsymbol{\beta}\}=[\mathbf{G}]\{\mathbf{F}\}$ as

$$
[\mathbf{C}]\{\boldsymbol{\beta}\}=[\mathbf{C}][\mathbf{G}]\{\mathbf{F}\},
$$

where $[\mathbf{G}]$ is concatenated flexibility matrix.

Combining (1) and (7), the governing equations of IFM can be symbolized as

$$
\left[\frac{[\mathbf{B}]}{[\mathbf{C}][\mathbf{G}]}\right]\{\mathbf{F}\}=\left\{\frac{\mathbf{P}}{\mathbf{0}}\right\}
$$

or

$$
[\mathbf{S}]\{\mathbf{F}\}=\left\{\mathbf{P}^{*}\right\}
$$

Thus both equilibrium and compatibility conditions are simultaneously satisfied in IFM.

$[\mathbf{B}]$ and $[\mathbf{G}]$ matrices of the structure are obtained by assembling the element equilibrium and flexibility matrices. Though the generation of elemental $[\mathbf{B}]$ and $[\mathbf{G}]$ matrices is straightforward, the computation of [C] needs considerable effort. The matrix [C] is not unique and its structure in terms of bandwidth, sparsity and computational effort is totally dependent on the scheme adopted in its generation. Recently, compatibility conditions have been classified and general procedures to generate them with optimum banded structure (Nagabhushanam \& Patnaik 1989) are being developed, which helps the use of efficient solution techniques for solving IFM governing equation.

\section{Nonlinear analysis by integrated force method}

Nonlinear structural behaviour is captured at the element level and the complete structure governing equations are established by enforcing equilibrium of forces and compatibility of displacements at the nodes. In the following, a total Lagrangian is used to develop elemental equilibrium matrix $\left[\mathbf{B}_{\mathbf{e}}\right]$ and elemental flexibility matrix $\left[\mathbf{G}_{\mathbf{e}}\right]$ for performing nonlinear analysis.

Displacement $\{\mathbf{u}\}$ and stress $\{\boldsymbol{\sigma}\}$ within the element are approximated in terms of two sets of independent variables as

$$
\begin{aligned}
& \{\mathbf{u}\}=\left[\mathbf{N}_{\mathbf{u}}\right]\left\{\mathbf{u}_{\mathbf{e}}\right\}, \\
& \{\boldsymbol{\sigma}\}=\left[\mathbf{N}_{\boldsymbol{\sigma}}\right]\left\{\mathbf{F}_{\mathbf{e}}\right\},
\end{aligned}
$$

where $\left\{\mathbf{u}_{\mathbf{e}}\right\}$ and $\left\{\mathbf{F}_{\mathbf{e}}\right\}$ are the nodal displacement vector and the generalised force parameter vector of the element respectively; $\left[\mathbf{N}_{\mathbf{u}}\right]$ and $\left[\mathbf{N}_{\boldsymbol{\sigma}}\right]$ are the matrices containing interpolation functions for displacement and stress fields respectively within the element.

The components of Green's strain vector $\{\boldsymbol{\varepsilon}\}$ in terms of the undeformed Lagrangian coordinates are given by the linear component $\left\{\varepsilon_{\mathbf{L}}\right\}$ and the nonlinear the component $\left\{\varepsilon_{\mathbf{N L}}\right\}$ which can be written as

$$
\{\boldsymbol{\varepsilon}\}=\left\{\boldsymbol{\varepsilon}_{\mathbf{L}}\right\}+\left\{\boldsymbol{\varepsilon}_{\mathbf{N L}}\right\} .
$$

Now $\left\{\varepsilon_{\mathbf{L}}\right\}$ can be expressed as

$$
\left\{\boldsymbol{\varepsilon}_{\mathbf{L}}\right\}=\left[\mathbf{D}_{\mathbf{f}}^{1}\right]\left[\mathbf{N}_{\mathbf{u}}\right]\left\{\mathbf{u}_{\mathbf{e}}\right\}=\left[\mathbf{B}_{\mathbf{L}}^{\mathbf{s}}\right]\left\{\mathbf{u}_{\mathbf{e}}\right\}
$$


where $\left[\mathbf{D}_{\mathbf{f}}^{1}\right]$ is a differential operator matrix and $\left[\mathbf{B}_{\mathbf{L}}^{\mathbf{s}}\right]=\left[\mathbf{D}_{\mathbf{f}}^{1}\right]\left[\mathbf{N}_{\mathbf{u}}\right]$, and $\left\{\boldsymbol{\varepsilon}_{\mathbf{N} \mathbf{L}}\right\}$ can be expressed as

$$
\left\{\boldsymbol{\varepsilon}_{\mathbf{N L}}\right\}=\frac{1}{2}\left[\mathbf{A}_{\boldsymbol{\theta}}\right]\{\boldsymbol{\theta}\},
$$

where $\{\boldsymbol{\theta}\}$ is the displacement gradient vector and $\left[\mathbf{A}_{\boldsymbol{\theta}}\right]$ contains the derivatives of the displacements. Further $\{\boldsymbol{\theta}\}$ can be expressed as

$$
\{\boldsymbol{\theta}\}=\left[\mathbf{D}_{\mathbf{f}}^{2}\right]\left[\mathbf{N}_{\mathbf{u}}\right]\left\{\mathbf{u}_{\mathbf{e}}\right\}=[\mathbf{G}]\left\{\mathbf{u}_{\mathbf{e}}\right\},
$$

where $\left[\mathbf{D}_{\mathbf{f}}^{2}\right]$ is a differential operator matrix and $[\mathbf{G}]=\left[\mathbf{D}_{\mathbf{f}}^{2}\right]\left[\mathbf{N}_{\mathbf{u}}\right]$.

Variation of strain is given by

$$
\begin{aligned}
& \{\mathrm{d} \varepsilon\}=\left\{\mathrm{d} \boldsymbol{\varepsilon}_{\mathbf{L}}\right\}+\left\{\mathrm{d} \boldsymbol{\varepsilon}_{\mathbf{N L}}\right\}, \\
& \{\mathrm{d} \varepsilon\}=\left(\left[\mathbf{B}_{\mathbf{L}}^{\mathbf{s}}\right]\left\{\mathbf{d} \mathbf{u}_{\mathbf{e}}\right\}+\frac{1}{2}\left[\mathbf{A}_{\boldsymbol{\theta}}\right][\mathbf{G}]\left\{\mathbf{d} \mathbf{u}_{\mathbf{e}}\right\}+\frac{1}{2}\left[\mathbf{d} \mathbf{A}_{\boldsymbol{\theta}}\right][\mathbf{G}]\left\{\mathbf{u}_{\mathbf{e}}\right\}\right), \\
& \{\mathrm{d} \boldsymbol{\varepsilon}\}=\left(\left[\mathbf{B}_{\mathbf{L}}^{\mathbf{s}}\right]+\left[\mathbf{A}_{\theta}\right][\mathbf{G}]\right)\left\{\mathbf{d} \mathbf{u}_{\mathbf{e}}\right\},
\end{aligned}
$$

since $\left[\mathrm{d} \mathbf{A}_{\theta}\right]\{\boldsymbol{\theta}\}=\left[\mathbf{A}_{\theta}\right]\{\mathrm{d} \boldsymbol{\theta}\}$. Expressing $\left[\mathbf{A}_{\boldsymbol{\theta}}\right][\mathbf{G}]=\left[\mathbf{B}_{\mathrm{NL}}^{\text {s }}\right]$, we can write (14) as

$$
\{\mathrm{d} \boldsymbol{\varepsilon}\}=\left[\mathbf{B}_{\mathbf{L}}^{\mathbf{s}}+\mathbf{B}_{\mathrm{NL}}^{\mathbf{s}}\right]\left\{\mathbf{d u}_{\mathbf{e}}\right\} .
$$

If $\{\overline{\mathbf{p}}\}$ denotes the surface forces per unit area of the deformed body $\bar{A}, \rho$ the mass density and $\{\mathbf{q}\}$ the body forces per unit mass, the Lagrangian virtual work expression over the volume $V$ for the virtual displacements $\{\mathrm{du}\}$ can be written as

$$
\int_{V}\{\mathrm{~d} \boldsymbol{\varepsilon}\}^{T} \sigma \mathrm{d} V=\int_{V} \rho\{\mathrm{d} \mathbf{u}\}^{T}\{\mathbf{q}\} \mathrm{d} V+\int_{A}\{\mathrm{~d} \mathbf{u}\}^{T}\left(\frac{\mathrm{d} \bar{A}}{\mathrm{~d} A}\{\bar{p}\}\right) \mathrm{d} A .
$$

Substituting (9) and (15) in (16) leads to

$$
\begin{aligned}
& \left\{\mathrm{du}_{\mathbf{e}}\right\}^{T} \int_{V}\left[\mathbf{B}_{\mathbf{L}}^{\mathbf{s}}+\mathbf{B}_{\mathrm{NL}}^{\mathbf{s}}\right]^{T}\left[\mathbf{N}_{\boldsymbol{\sigma}}\right]\left\{\mathbf{F}_{\mathbf{e}}\right\} \mathrm{d} V \\
& \quad=\left\{\mathrm{d} \mathbf{u}_{\mathrm{e}}\right\}^{T}\left[\int_{V} \rho\left[\mathbf{N}_{\mathbf{u}}\right]^{T}\{\mathbf{q}\} \mathrm{d} V+\int_{A}\left[\mathbf{N}_{\mathbf{u}}\right]^{T}\left(\frac{\mathrm{d} \bar{A}}{\mathrm{~d} A}\{\bar{p}\}\right) \mathrm{d} A\right] .
\end{aligned}
$$

Let the right hand side of (17) be represented by $\left\{\mathbf{P}_{\mathbf{e}}\right\}$ which is a vector of equivalent nodal forces due to the body forces $\{\mathbf{q}\}$ and surface tractions $\{\overline{\mathbf{p}}\}$. Then the nonlinear equilibrium equation becomes:

$$
\int_{V}\left[\mathbf{B}_{\mathbf{L}}^{\mathbf{s}}+\mathbf{B}_{\mathbf{N L}}^{\mathbf{s}}\right]^{T}\left[\mathbf{N}_{\boldsymbol{\sigma}}\right]\left\{\mathbf{F}_{\mathbf{e}}\right\} \mathrm{d} V=\left\{\mathbf{P}_{\mathbf{e}}\right\}
$$

The unbalanced load $\psi$ will be

$$
\begin{aligned}
& \{\boldsymbol{\psi}\}=\int_{V}\left[\mathbf{B}_{\mathbf{L}}^{\mathbf{s}}+\mathbf{B}_{\mathbf{N L}}^{\mathbf{s}}\right]^{T}\left[\mathbf{N}_{\boldsymbol{\sigma}}\right]\left\{\mathbf{F}_{\mathbf{e}}\right\} \mathrm{d} V-\left\{\mathbf{P}_{\mathbf{e}}\right\}, \\
& \{\boldsymbol{\psi}\}=\left[\mathbf{B}_{\mathbf{e}}\right]\left\{\mathbf{F}_{\mathbf{e}}\right\}-\left\{\mathbf{P}_{\mathbf{e}}\right\},
\end{aligned}
$$

where $\left[\mathbf{B}_{\mathbf{e}}\right]$ is the elemental equilibrium matrix and can be expressed as

$$
\left[\mathbf{B}_{\mathbf{e}}\right]=\int_{V}\left[\mathbf{B}_{\mathbf{L}}^{\mathbf{s}}+\mathbf{B}_{\mathbf{N L}}^{\mathbf{s}}\right]^{T}\left[\mathbf{N}_{\sigma}\right] \mathrm{d} V
$$


The relation between the elemental stress parameters of the element and the generalized deformations $\left\{\boldsymbol{\beta}_{\mathbf{e}}\right\}$ are established through the complementary energy principle.

Considering complimentary strain energy, we can write

$$
\int_{V}\{\mathrm{~d} \sigma\}^{T}\{\epsilon\} \mathrm{d} V=\left\{\mathrm{d} F_{e}\right\}^{T}\left\{\beta_{e}\right\}
$$

where $\left\{\boldsymbol{\beta}_{\mathrm{e}}\right\}$ represents the generalised element deformation vector. With stress strain relationship $\{\varepsilon\}=[\phi]\left\{\sigma_{\mathbf{e}}\right\}$ where $[\phi]$ is the compliance matrix, (21) can be written as

$$
\begin{aligned}
& \int_{V}\left\{\mathrm{~d} \mathbf{F}_{\mathbf{e}}\right\}^{T}\left[\mathbf{N}_{\sigma}\right]^{T}[\phi]\left[\mathbf{N}_{\boldsymbol{\sigma}}\right]\left\{\mathbf{F}_{\mathbf{e}}\right\} \mathrm{d} V=\left\{\mathbf{d F}_{\mathbf{e}}\right\}^{T}\left\{\boldsymbol{\beta}_{\mathbf{e}}\right\}, \\
& \left\{\mathrm{d} \mathbf{F}_{\mathbf{e}}\right\}^{T}\left(\int_{V}\left[\mathbf{N}_{\boldsymbol{\sigma}}\right]^{T}[\phi]\left[\mathbf{N}_{\boldsymbol{\sigma}}\right]\left\{\mathbf{F}_{\mathbf{e}}\right\} \mathrm{d} V-\left\{\boldsymbol{\beta}_{\mathbf{e}}\right\}\right)=0 .
\end{aligned}
$$

Equation (22) leads to

$$
\left\{\boldsymbol{\beta}_{\mathbf{e}}\right\}=\left[\mathbf{G}_{\mathbf{e}}\right]\{\mathbf{F}\},
$$

where

$$
\left[\mathbf{G}_{\mathbf{e}}\right]=\int_{V}\left[\mathbf{N}_{\boldsymbol{\sigma}}\right]^{T}[\boldsymbol{\phi}]\left[\mathbf{N}_{\boldsymbol{\sigma}}\right] \mathrm{d} V .
$$

The developed $\left[\mathbf{B}_{\mathbf{e}}\right]$ and $\left[\mathbf{G}_{\mathbf{e}}\right]$ are utilized to generate $[\mathbf{B}],[\mathbf{G}]$ and $[\mathbf{C}]$ of the structure. Subsequently the IFM's governing equation for a nonlinear structure becomes

$$
\psi(\mathbf{a})=\left[\frac{[\mathbf{B}]}{[\mathbf{C}][\mathbf{G}]}\right]\{\mathbf{F}\}-\left\{\frac{\mathbf{P}}{\mathbf{0}}\right\}
$$

where (a) corresponds to the deformed geometry of the structures at the given load step.

The solution to this equation is achieved using the Newton-Raphson method with load increments. Iterations within a load increment will continue until the residual load $\psi$ satisfies the convergence criteria.

\section{Development of elemental matrices}

For analysing nonlinear beam bending problems, three different types of element formulations are considered. They are the classical two-node 2D beam element (BEAM2D), eightnode rectangular isoparametric element (ISPM08-13) and six-node paralinear isoparametric element (ISPM06-9).

The idealized BEAM2D element is shown in figure 1a. The displacement shape functions are Hermite polynomials, which are

$$
\left\{\begin{array}{l}
\mathbf{u} \\
\mathbf{v}
\end{array}\right\}=\left[\begin{array}{cccccc}
H_{1} & 0 & 0 & H_{4} & 0 & 0 \\
0 & H_{2} & l_{e} H 3 & 0 & H_{5} & l_{e} H_{6}
\end{array}\right]\left\{\mathbf{u}_{\mathbf{n}}\right\}
$$

where $H_{i}$ are Hermite polynomials expressed in normalized coordinate $\xi,\left(\xi=x / l_{e}\right)$,

$$
\begin{array}{ll}
H_{1}=1-\xi, & H_{4}=\xi \\
H_{2}=1-3 \xi^{2}+2 \xi^{3}, & H_{5}=3 \xi^{2}-2 \xi^{3}, \\
H_{3}=\xi-2 \xi^{2}+\xi^{3} & H_{6}=-\xi+\xi^{3} .
\end{array}
$$


(a)
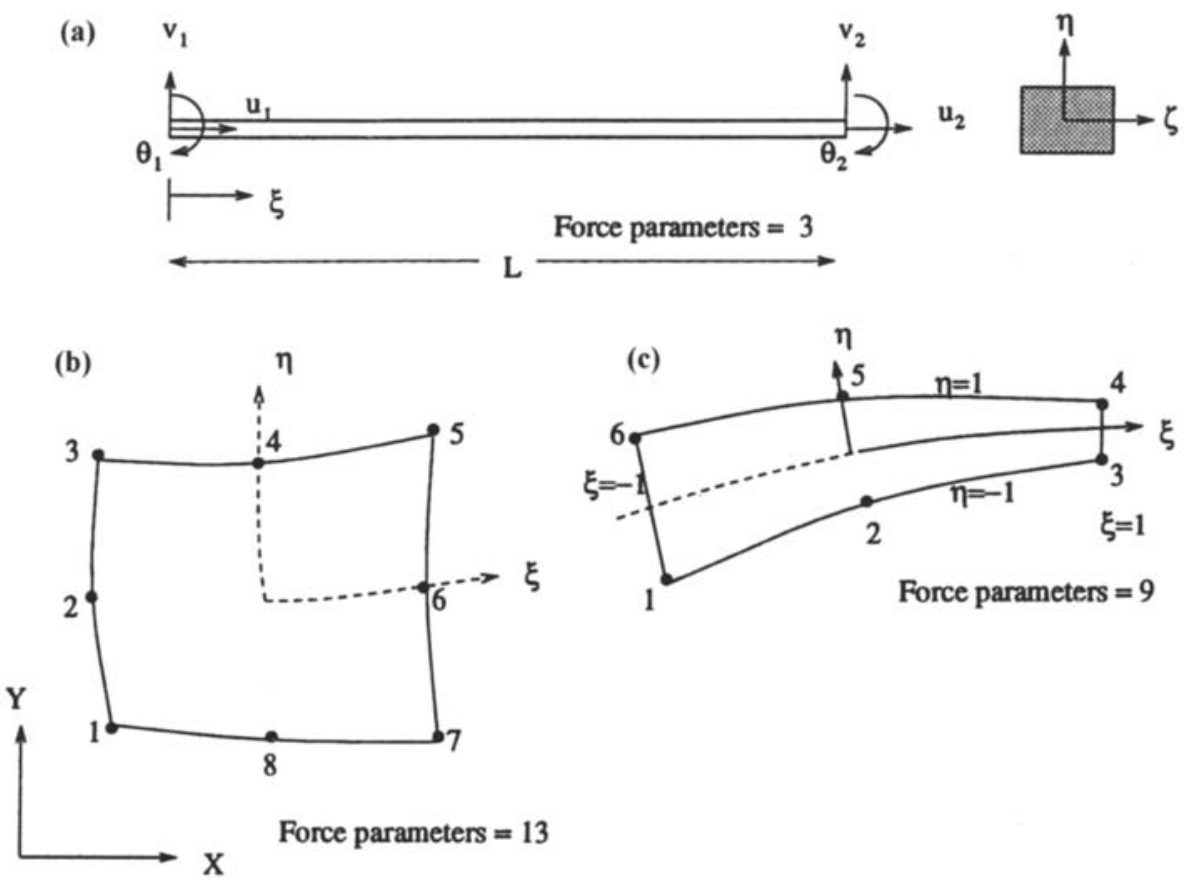

Figure 1. Elements developed for nonlinear analysis using IFM: (a) BEAM2D element, (b) ISPM08-13 element, (c) ISPM06-9 element.

Axial stress at any point $\eta ; \xi$ of the beam can be written as

$$
\{\boldsymbol{\sigma}\}=\left[\mathbf{N}_{\boldsymbol{\sigma}}\right]\left\{\mathbf{F}_{\mathbf{e}}\right\}=\left[\begin{array}{lll}
1 & \eta & \eta \xi
\end{array}\right]\left\{\begin{array}{l}
F_{1} \\
F_{2} \\
F_{3}
\end{array}\right\},
$$

where $F_{1}, F_{2}$ and $F_{3}$ are the force parameters of the beam element.

For the eight-node isoparametric element (ISPM08-13) seen in figure $1 \mathrm{~b}$, the shape functions used for corner nodes are

$$
N_{i}=\frac{1}{4}\left(1+\xi_{0}\right)\left(1+\eta_{0}\right)\left(\xi_{0}+\eta_{0}-1\right)
$$

and for mid-side nodes

$$
N_{i}=\frac{\xi_{i}^{2}}{2}\left(1+\xi_{0}\right)\left(1-\eta^{2}\right)+\frac{\eta_{i}^{2}}{2}\left(1+\eta_{0}\right)\left(1-\xi^{2}\right),
$$

where $\xi_{0}=\xi \xi_{i}$ and $\eta_{0}=\eta \eta_{i}$.

The stress field within the element is assumed in terms of 13 independent force parameters $\sigma_{1}, \sigma_{2} \cdots \sigma_{13}$ as given below.

$$
\begin{aligned}
\sigma_{x} & =\sigma_{1}+\sigma_{2} y+\sigma_{6} x+\sigma_{8} x^{2}+\sigma_{10} y^{2}-2 \sigma_{13} x y, \\
\sigma_{y} & =\sigma_{3}+\sigma_{4} x+\sigma_{7} y+\sigma_{9} x^{2}+\sigma_{11} y^{2}-2 \sigma_{12} x y, \\
\sigma_{x y} & =\sigma_{5}-\sigma_{6} y-\sigma_{7} x-2\left(\sigma_{10}+\sigma_{11}\right) x y+\sigma_{12} x^{2}+\sigma_{13} y^{2} .
\end{aligned}
$$

The assumed stress field satisfies the stress equilibrium within the element. 
For the six-node paralinear isoparametric element (ISPM06-9) (fig 1c), the shape functions used for corner nodes are

$$
N_{i}=\frac{1}{4}\left(\xi_{0}+\xi^{2}\right)\left(1+\eta_{0}\right)
$$

and for mid-side nodes

$$
N_{i}=\frac{1}{2}\left(1-\xi^{2}\right)\left(1+\eta_{0}\right),
$$

where $\xi_{0}=\xi \xi_{i}$ and $\eta_{0}=\eta \eta_{i}$.

A stress distribution with nine independent force parameters $\sigma_{1}, \sigma_{2} \cdots \sigma_{9}$ that satisfies the homogeneous stress equilibrium conditions within the element is considered as follows.

$$
\begin{aligned}
\sigma_{x} & =\sigma_{1}+\sigma_{2} y+\sigma_{6} x-2 \sigma_{8} x y, \\
\sigma_{y} & =\sigma_{3}+\sigma_{4} x+\sigma_{7} y-2 \sigma_{9} x y, \\
\sigma_{x y} & =\sigma_{5}-\sigma_{6} y-\sigma_{7} x+\sigma_{8} x^{2}+\sigma_{9} x^{2} .
\end{aligned}
$$

The elemental $\left[\mathbf{B}_{\mathbf{e}}\right]$ and $\left[\mathbf{G}_{\mathbf{e}}\right]$ are calculated by considering (20) and (24) respectively. The Gaussian quadrature technique is utilized to perform the integration in these equations.

\section{Results}

A number of highly nonlinear structures are considered and each one of them is solved using the proposed elements. The results obtained by IFM are compared either with analytical solutions or with those of the displacement method to establish the viability of effectively utilizing IFM for nonlinear structural analysis.

\subsection{Cantilever beam with end moment}

The highly nonlinear behaviour of a cantilever beam with end moment was analysed by Surana (1983) using the displacement method with curved beam elements with mid-node. The same cantilever beam, whose geometry, structural details and loading is shown in figure 2 , is analysed by IFM. The cantilever beam is subjected to an end moment $M=m f$ ( $f$ is varied from 0 to 2 ) and the total moment is applied in 20 equal increments. At each load increment, equilibrium iterations are performed until convergence is within the tolerance limits. The tolerance limit is fixed such that the successive displacements of any two iterations should not vary by more than $0.0001 \%$.

The same structure which is idealised as above is also analysed by the displacement method (DM) using straight beam elements. It was observed that the displacement method generally took 5 iterations at each load step, while the IFM requires only two iterations. Thus IFM seems to have faster convergence when compared to the displacement method for this problem. Figure 3 shows load factor $f$ vs tip deflections $\delta_{x}$ and $\delta_{y}$, which are from those obtained by IFM, and the displacement method (DM), and those given by Surana (1983). The results from all three methods are very close to one another.

The results of the deformed geometry of the beam obtained by IFM are compared with the results of Surana (1983) as the load factor $f$ is increased and they are shown in figure 4. It can be seen that there is close agreement between them for all load factors. Thus IFM, when extended to perform nonlinear analysis, is able to predict the nonlinear behaviour of load vs deflections as effectively as the displacement method. 

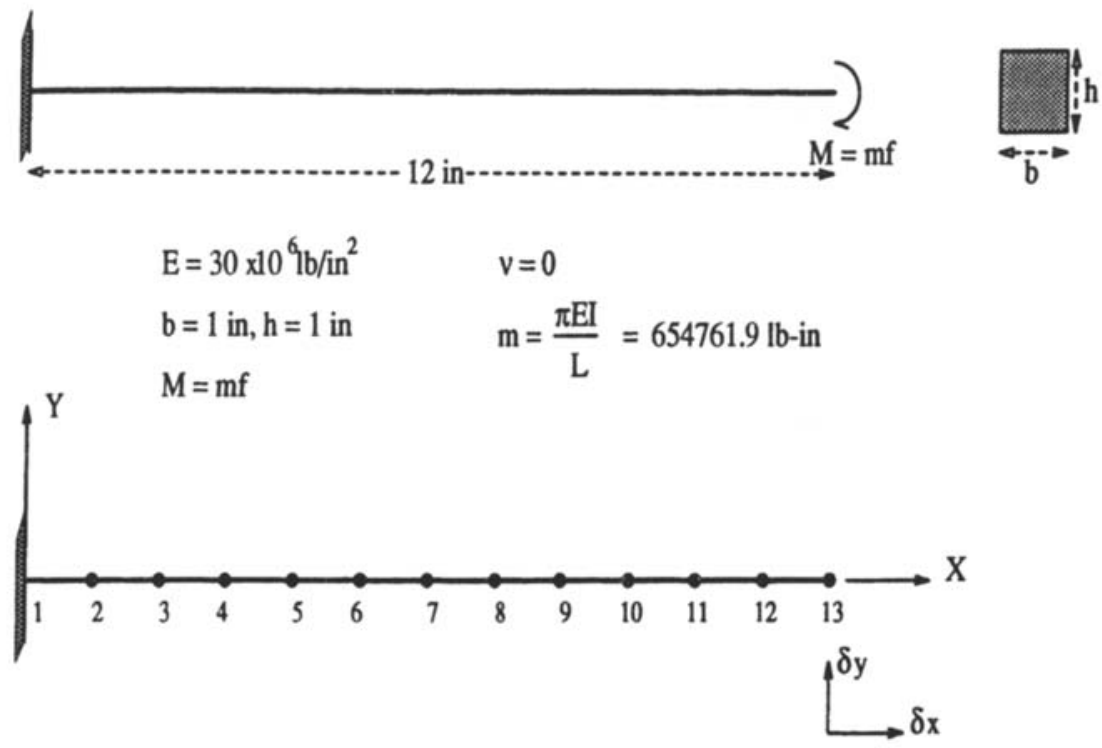

Figure 2. Cantilever beam with the end moment, finite element model with beam elements.

\subsection{Plane stress cantilever beam}

A plane stress cantilever beam with a concentrated load applied at the free end as shown in figure 5 is modelled with five 8-node isoparametric elements. The cantilever beam is analysed using IFM and compared with the results of displacement method (Nayak 1980).

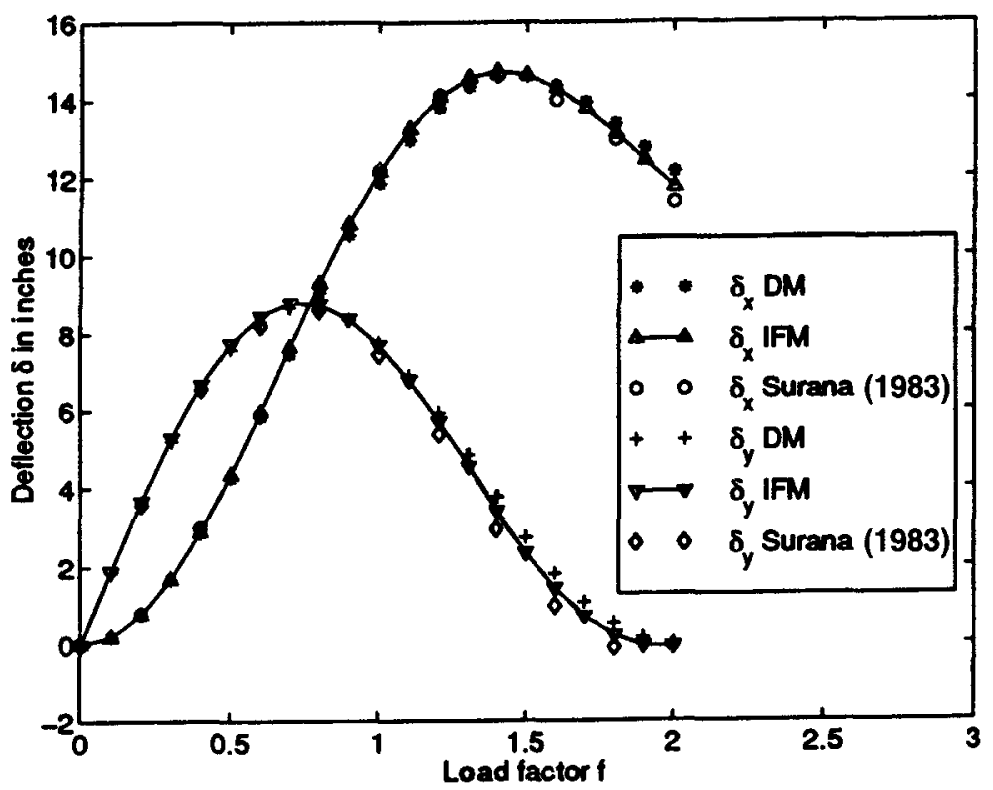

Figure 3. Load-deflection plots for cantilever beam with end moment. 


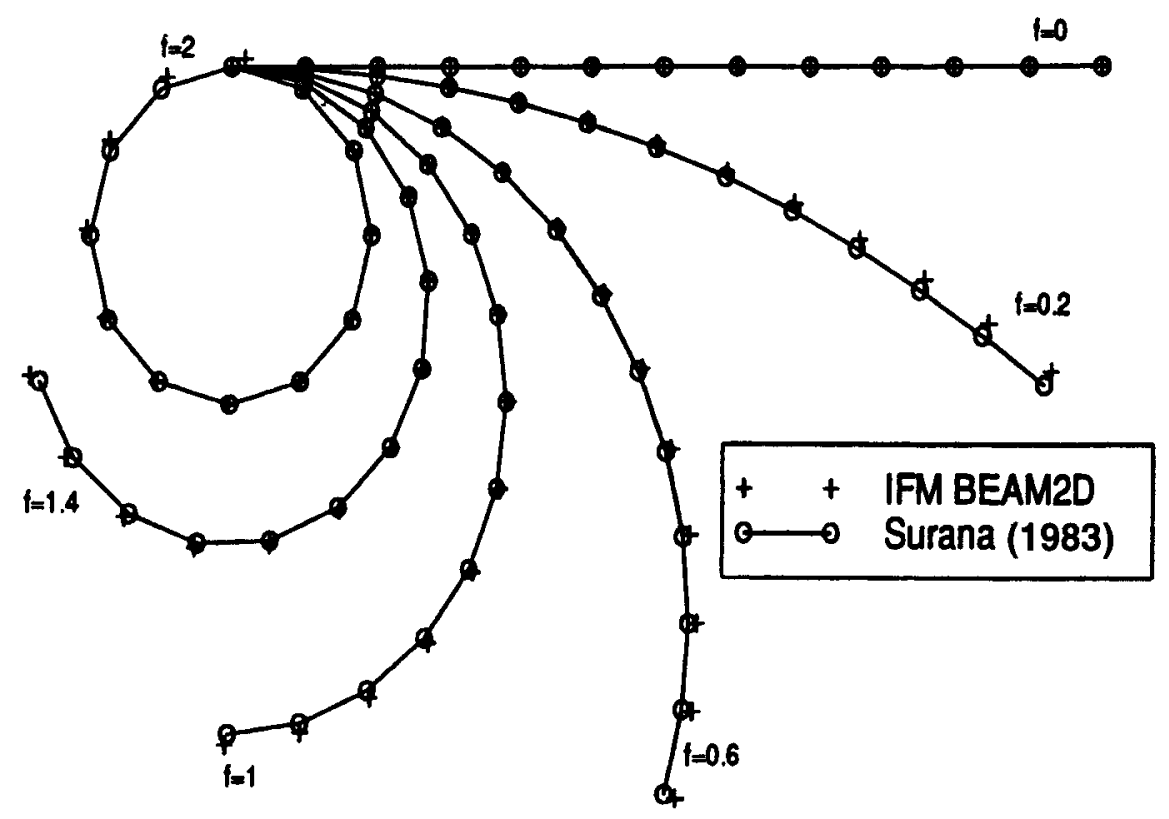

Figure 4. Deformed shapes of the cantilever beam with end moment.

The deflections at the load application $\delta_{x}$ and $\delta_{y}$ are plotted against the non-dimensional load factor $f\left(P L^{2}\left(1-\nu^{2}\right) / E I\right)$ for $\nu=0.0$ and 0.3 and are shown in figure 6 . The curves show that the results predicted by the IFM and the displacement method are extremely close to each other.

\subsection{Portal frame}

A portal frame is considered, whose geometry, structural details, and loading are given in figure 7. Half of the structure is considered and is idealised with sixteen elements. The structure is analysed using IFM with BEAM2D, ISPM08-13 and ISPM06-9 elements. These results are compared with those obtained by the displacement method using curved beam elements given by Surana (1983), and are shown in figures 8 and 9. Figure 8 shows the behaviour of non-dimensional horizontal displacement $\delta_{H}^{B} / L$ at point $\mathrm{B}$ against the non-

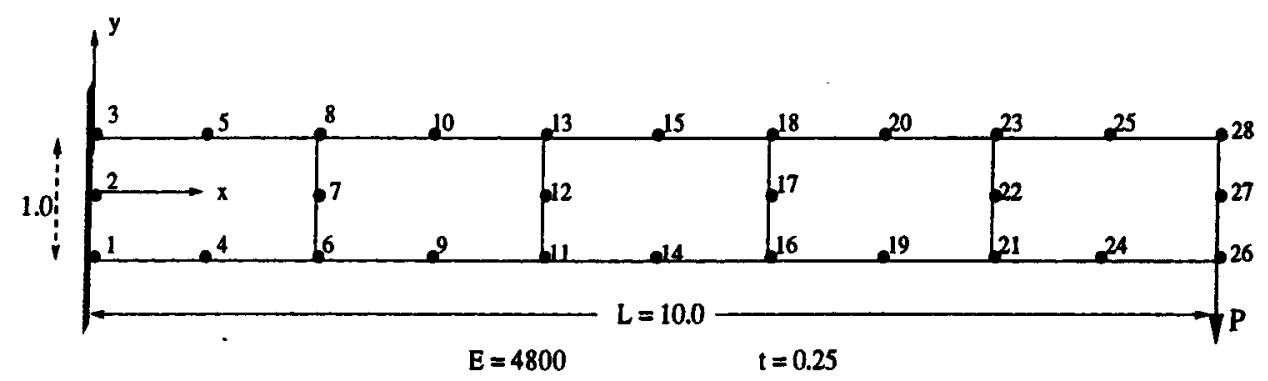

Figure 5. Cantilever beam with concentrated load, modelled with ISPM08-13 elements. 


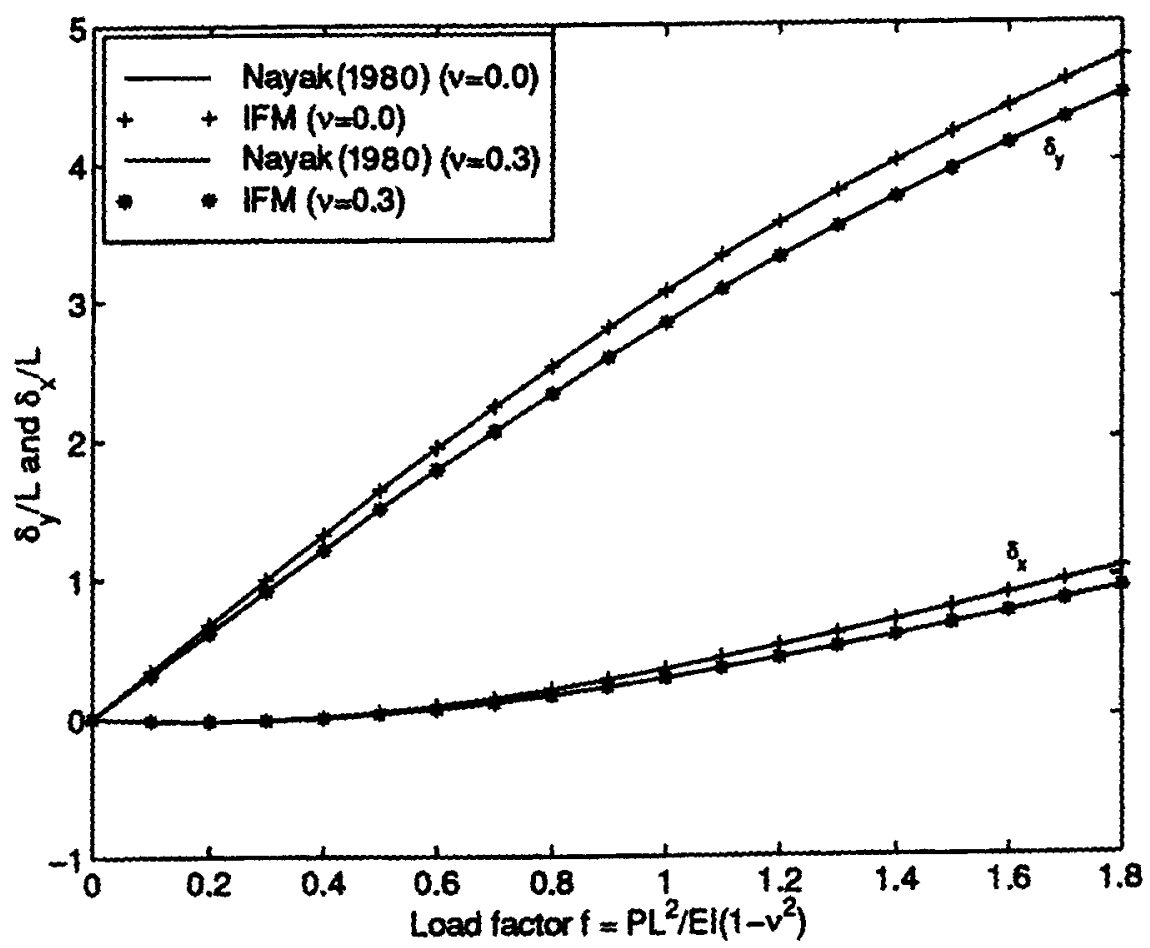

Figure 6. Load-deflection plots for cantilever beam with concentrated tip load.

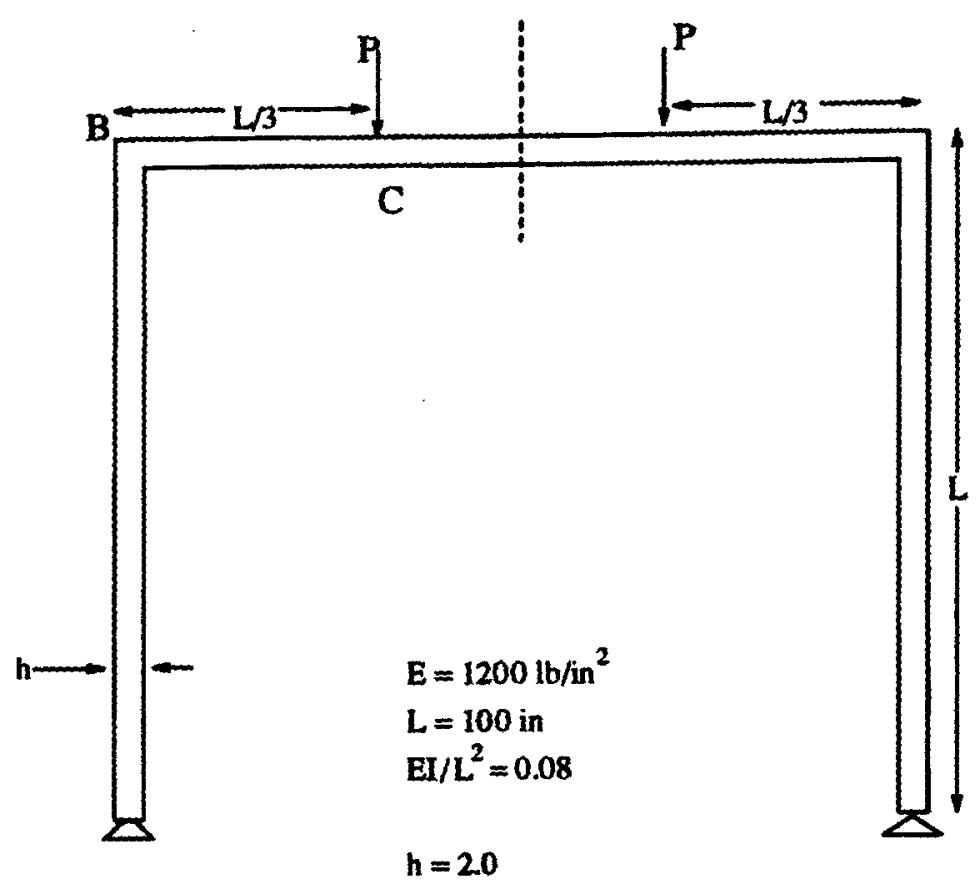

Figure 7. Portal frame with symmetric loading. 


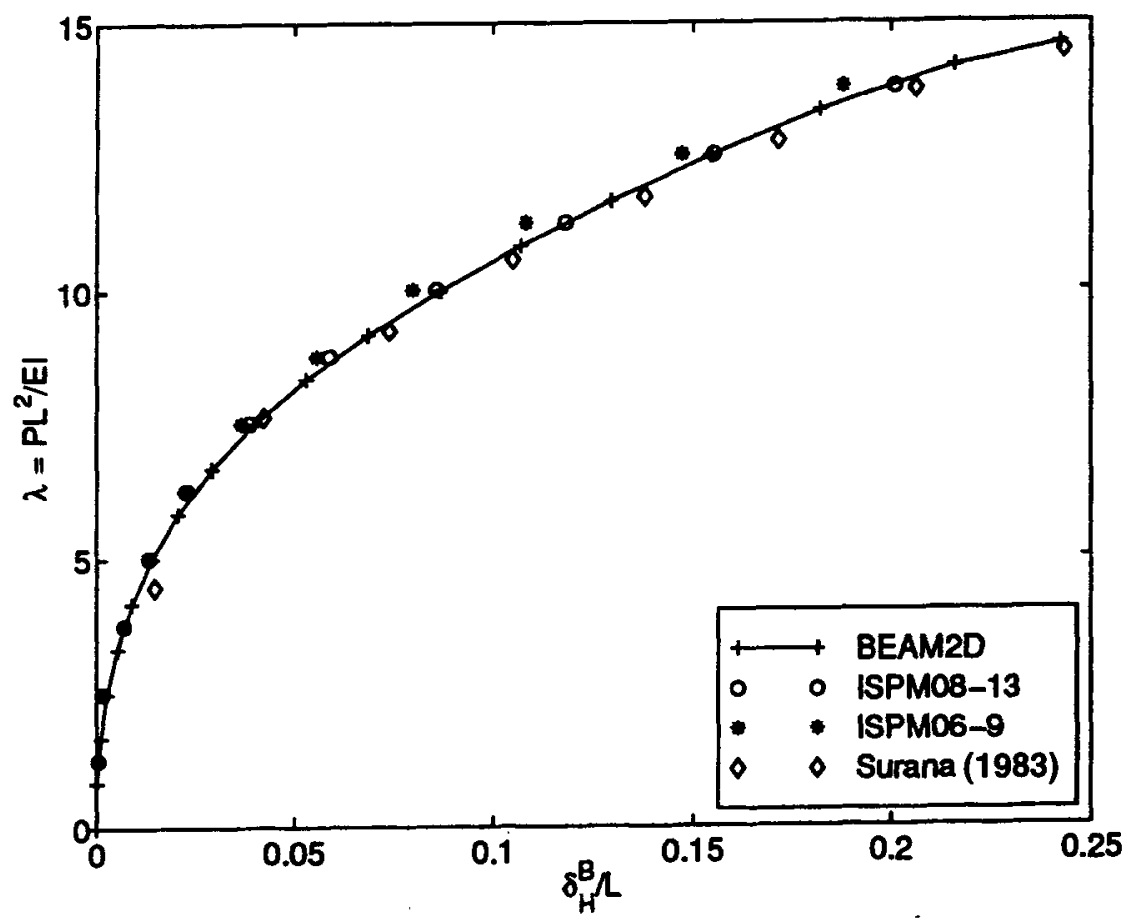

Figure 8. Load-deflection characteristics of the portal frame.

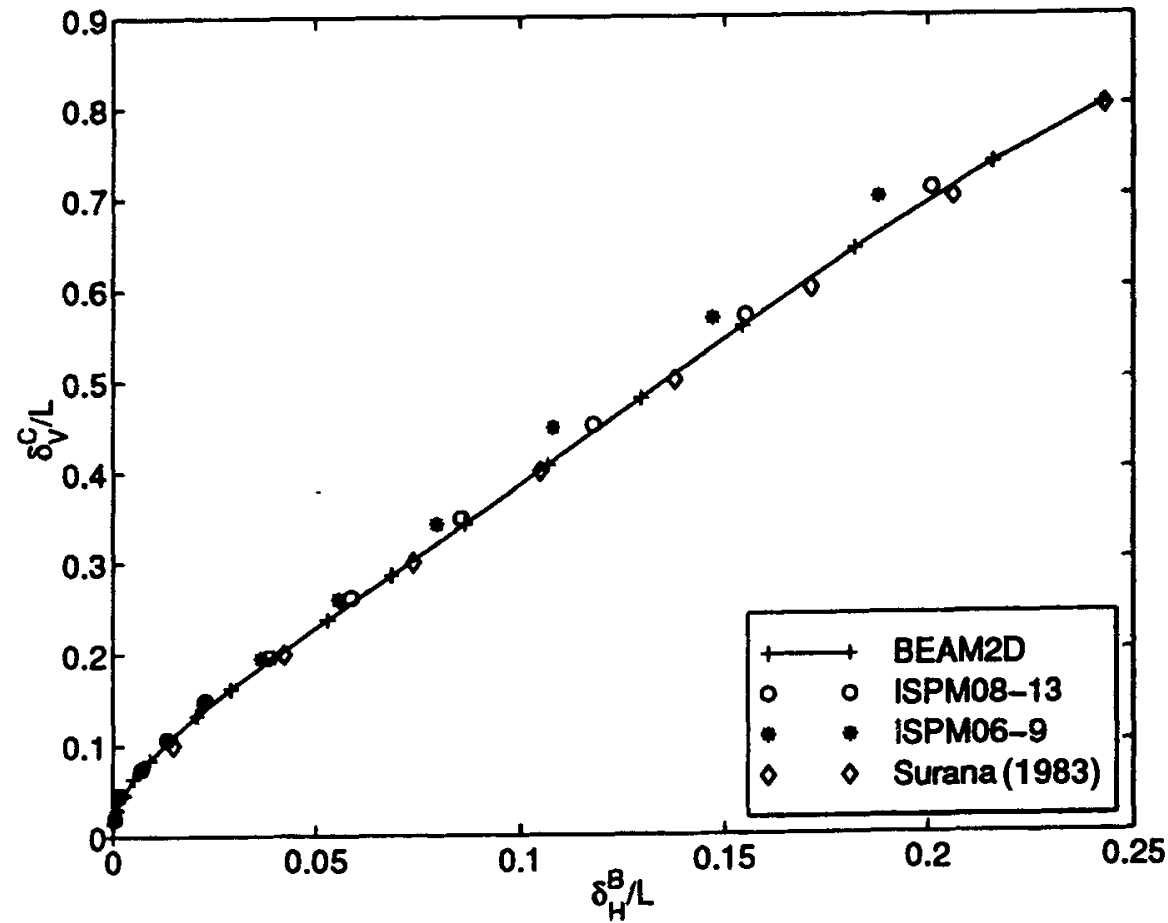

Figure 9. Load-deflection characteristics of the portal frame. 


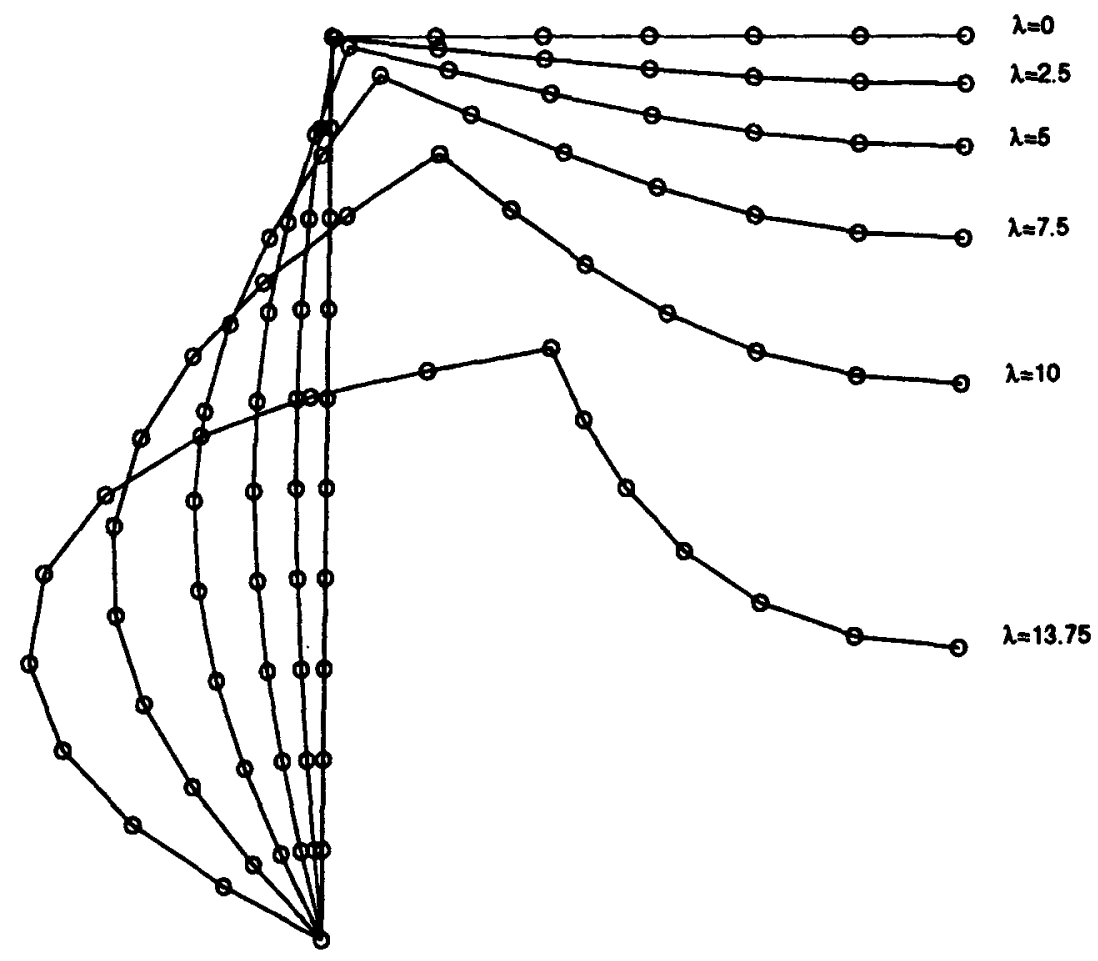

Figure 10. Deformed shape of the portal frame for various load factors.

dimensional load parameter $\lambda=P L^{2} /$ EI. The non-dimensional horizontal displacement at point $\mathrm{B}, \delta_{H}^{B} / L$, is plotted against the non-dimensional vertical displacement $\delta_{V}^{C} / L$ at point $\mathrm{C}$ and is shown in figure 9. Among the results of various elements, results of ISPM08-13 are close to the results of the displacement method with curved beam elements.

The deformed shape of the portal frame as computed by IFM using ISPM08-13 for various load factors is shown in figure 10. It clearly exposes the highly nonlinear behaviour of the portal frame for the load range considered.

\section{Conclusions}

Application of integrated force method for analysing nonlinear structures is presented for the first time. A general formulation to develop the elemental equilibrium and flexibility matrices is described. Three representative highly nonlinear beam-type structures are considered and they are analysed using beam and plane stress elements. The results of the integrated force method are compared with those of the displacement method. It shows that the integrated force method can be successfully applied to highly nonlinear structures and is also a viable alternative method for the currently widely used displacement method.

\section{References}

Kaljevic I, Patnaik S N, Hopkins D A 1996a Development of finite elements for two-dimensional structural analysis using integrated force method. Comput. Struct. 59: 691-706 
Kaljevic I, Patnaik S N, Hopkins D A 1996b Three-dimensional structural analysis by the integrated force method. Comput. Struct. 58: 869-886

Kaneko I, Lawo H, Thierauf G 1983 On computational procedures for the force method. Int. J. Numer. Methods Eng. 18: 1469-1495

Nagabhushanam J, Patnaik S N 1989 General purpose program to generate compatibility matrix for the integrated force method. AIAA J. 28: 1838-1842

Nagabhushanam J, Srinivas C J 1991 Automatic generation of sparse and banded compatibility matrix for the integrated force method. Comput. Mechanics ' 91 , International Conference on Computing in Engineering Science, Patras, Greece, pp. 20-25

Nayak G C 1980 Finite deformations by finite elements. Proceedings of the Symposium on Large Deformations in memory of Prof B Karunes, pp. 413-429

Oden J T, Neighbors A 1976 Network-topological formulation of analysis of geometrically and materially non-linear space frames. Proc. Ist Int. Conf. Space Structures (ed.) R M Davis (London: Blackwell Scientific)

Patnaik S N 1973 An integrated force method for discrete analysis Int. J. Numer. Methods Eng. 16: 236-251

Patnaik S N 1986 The variational energy formulation for the integrated force method. AIAA J. 24: 129-137

Patnaik S N, Yadagiri S 1976 Frequency analysis of structures. Comput. Methods Appl. Mech. Eng. 9: 245-265

Patnaik S N, Hopkins D A, Coroneos R 1996 Structural optimization with approximate sensitivities. Comput. Struct. 58: 407-418

Robinson J, Haggenmacher G W 1971 Some new developments in matrix force analysis. In Recent advances in matrix methods of structural analysis and design (Univ. Alabama) pp. 183-228

Surana K S 1983 Geometrically non-linear formulation for two dimensional curved beam elements. Comput. Struct. 17: 105-114

Topou A 1979 A contribution to the systematic analysis of finite element structures using the force method (in German). Doctoral dissertation, Essen University, Essen, Germany 